\title{
Path Integral Calculations of exchange in solid ${ }^{4} \mathrm{He}$
}

\author{
B. Bernu \\ LPTL, UMR 7600 of CNRS, Université P. et M. Curie, Paris, France \\ D. M. Ceperley \\ Dept. of Physics and NCSA, University of Illinois at Urbana-Champaign, Urbana, IL 61801
}

\begin{abstract}
Recently there have been experimental indications that solid ${ }^{4} \mathrm{He}$ might be a supersolid. We discuss the relation of supersolid behavior to ring exchange. The tunnelling frequencies for ring exchanges in quantum solids are calculated using Path Integral Monte Carlo by finding the free energy for making a path that begins with the atoms in one configuration and ends with a permutation of those positions. We find that the exchange frequencies in solid ${ }^{4} \mathrm{He}$ are described by a simple lattice model which does not show supersolid behavior. Thus, the PIMC calculations constrain the mechanism for the supersolid behavior. We also look at the characteristics of very long exchanges needed for macroscopic mass transport.
\end{abstract}

Key words: supersolid, solid ${ }^{4} \mathrm{He}$, Path Integral Monte Carlo

PACS: 67.80.-s, 02.70.Ss, 05.30.Jp

Recent torsional-oscillator observations by E. Kim and M. H. W. Chan on solid ${ }^{4} \mathrm{He}$, both in the disordered absorbed vycor and in bulk ${ }^{4} \mathrm{He}[1]$, have revived interest in the super-solid phase. A supersolid[2] is characterized by both long-range translational order and by a non-classical response to rotation (NCRI). In attempting to understand these results, one should first assume that the observed phenomena is described by equilibrium thermodynamics of pure bulk solid helium. For such a model, it is possible to perform numerically exact Path Integral Monte Carlo simulations and explore its basic properties. If such properties agree with experiments, that will provide strong

Email address: ceperley@uiuc.edu (D. M. Ceperley). support for the model. If one finds disagreement, then the basic assumption is invalid. The results of such a calculation have been recently published[3]. Here, we elaborate and update that publication.

We consider the possibility that bulk solid hcp helium, assumed to be free of defects, could have an equilibrium supersolid phase. Because of its light mass, solid helium has large quantum zero point fluctuations causing instantaneous ground state defects. At any instant of time, a good fraction of atoms are closer to a neighboring site than to their home site. However, the absence of an atom from a lattice site is not sufficient for having a supersolid; if the empty site is always accompanied by doubly occupied site, there can be no mass current. However, Chester[4] proved that any Jastrow 
(i.e. pair product) wavefunction of finite range has both BEC and vacancies. Although Jastrow wavefunctions do not make a good description of the ground state, one can multiply by a periodic trial function $[5,6]$ and achieve a crystalline, Bose condensed state and a reasonable description of the energy, though not as low as a wavefunction without BEC. Though this wavefunction of the solid is not too bad for static properties, other properties, particularly off-diagonal long range order (ODLRO), equivalent to BEC, is not necessarily accurately given. There is no reason to think that even a perfect match of the diagonal properties constrains the off-diagonal properties.

Path integrals give a rigorous way of calculating on and off-diagonal properties. The partition function of $N$ bosons is:

$Z=\frac{1}{N !} \sum_{P} \int d R\left\langle R\left|e^{-\beta H}\right| P R\right\rangle$

where $H$ is the Hamiltonian, $\beta$ the inverse temperature and $R=\left\{\mathbf{r}_{1}, \mathbf{r}_{2}, \ldots \mathbf{r}_{N}\right\}$. We will assume the helium atoms interact with a semi-empirical[7] pair potential. The helium potential is known to be accurate; many calculations agree with experimental measurements, without the adjustments needed in all other atomic and molecular systems. Using the PIMC method, superfluidity and freezing, happen naturally at the right density and temperature, without imposing them in any way as is done in a wavefunction based approach.

To perform the PIMC calculations, the density matrix operator is expanded into a path, $R(t)$ where $\mathrm{t}(0 \leq t \leq \beta)$ is imaginary time. Then the superfluid density (i.e. the number of atoms not moving with the walls of the apparatus) is proportional to the mean squared winding number, $\rho_{s} \propto<\vec{W}^{2}>$ where $\vec{W}=\int_{0}^{\beta} d t \sum_{i=1}^{N} d \mathbf{r}_{i}(t) / d t$ is the winding number of the paths around the periodic boundary condition. Note that only exchanges on the order of the sample size contribute to the superfluid density; local exchanges make no contribution. Prokof'ev and Svistunov[8] argue that long windings are impossible without vacancies and interstitials that are spatially separated.

The technical complications of path integral calculations[9] concern ergodicity of the random walk, and finite size effects: one has to take the limit as $N \rightarrow \infty$. If one does a PIMC simulation of a 48 atom $(3 \times 4 \times 2)$ hcp supercell in $\mathrm{PBC}$, one finds a superfluid density of about $3 \%$ at melting density (molar volume $21.04 \mathrm{~cm}^{3}$ ) and about $1.2 \%$ at 55 bars (molar volume $19.01 \mathrm{~cm}^{3}$ ). However, simulations of a larger cell 180 atoms, show zero superfluid density. With current algorithms, it is difficult to be sure that the lack of winding paths has a physical origin, or is due to a lack of ergodicity within the random walk. Winding number changes involve the permutation of a group of particles which stretch across the box. One needs to update simultaneously 5 atom coordinates to change the winding number in the 180 cell. The acceptance probability for such a collective move becomes very small. To avoid the problem of estimating rare events, we turn to a PIMC approach which directly estimates individual exchange probabilities.

The Thouless[10] theory of exchange in quantum crystals assumes that at low temperatures, the system will almost always be near one of the $N$ ! arrangements of particles to lattice sites. There will be rare, rapid, tunnellings from one arrangement to another in an amount of "imaginary time" $\gamma_{p}$. Hence, we can label the particles with their initial lattice sites and drop the $N$ ! in Eq. (1). The partition function is written as a sum over permutations of lattice sites onto themselves. We break up the permutation into cyclic exchanges $P \equiv$ $\left\{p_{1}, p_{2} \ldots p_{n}\right\}$. We can then write:

$Z=Z_{0} \sum_{P} \prod_{i=1}^{n_{P}} f_{p_{i}}(\beta)$.

where $Z_{0}=\int d R\left\langle R\left|e^{-\beta H}\right| R\right\rangle$ is an uninteresting phonon partition function at low temperature and the contribution for an elementary cycle is:

$f_{p}(\beta)=\frac{1}{Z_{0}} \int d R\left\langle R\left|e^{-\beta H}\right| p R\right\rangle \equiv J_{p} \beta$.

The exchange is localized in imaginary time so the path integral is proportional to the number of crossing times: $f_{p}(\beta) \propto \beta$; the coefficient, $J_{p}$ is the exchange frequency. We have also assumed that independent cycles do not interfere with each other, reasonable because the exchanges are rare and are 
Table 1

Calculated exchange frequencies in hcp ${ }^{4} \mathrm{He}$ at a molar volume of $21.04 \mathrm{cc}$ in units of $\mu K$. The notation of the exchange is from Roger[13] as are the WKB actions for ${ }^{3} \mathrm{He}$. The column $J_{48}$ are for $\beta=1, \tau=0.0125$ and $N=48$ and $N=180$ for a larger cell. $\gamma_{p}$ is the exchange time in units of $K^{-1}$.

\begin{tabular}{|c|c|ccc|cc|c|c|}
\hline $\mathrm{p}$ & name & $J_{48}$ & $\%$ & error & $J_{180}$ & error & $\gamma_{p}$ & WKB \\
\hline 2 & $\mathrm{nn}$ & 1.7 & 2 & 3.74 & 7 & 0.32 & 9.54 \\
& $\mathrm{nn}$ & 2.5 & 6 & 4.50 & 5 & 0.31 & 8.60 \\
\hline 3 & $\mathrm{~T}$ & 5.6 & 4 & 8.0 & 5 & 0.29 & 7.33 \\
& $\mathrm{~T}^{*}$ & 0.62 & 5 & 1.25 & 6 & 0.34 & 8.83 \\
& $\mathrm{~T}^{\prime}$ & 2.0 & 4 & 4.9 & 5 & 0.32 & 7.75 \\
\hline 4 & $\mathrm{Kp}$ & 0.74 & 9 & 0.59 & 6 & 0.19 & 9.00 \\
& $\mathrm{Kp}$ & 0.84 & 8 & 0.71 & 6 & 0.4 & 9.38 \\
& $\mathrm{Ksq}$ & 1.25 & 4 & 2.2 & 8 & 0.34 & 8.09 \\
\hline
\end{tabular}

unlikely to be close in both space and imaginary time.

We have developed methods[11,12] to calculate the exchange frequencies in quantum crystals, primarily for solid ${ }^{3} \mathrm{He}$. In that system, ring exchanges of 2-6 atoms give rise to the magnetic properties at low temperatures. The PIMC calculations were important in giving a microscopic justification of the multiple exchange model. The PIMC values are in good agreement with experimental data on ${ }^{3} \mathrm{He}$ and are thus expected to be accurate in the closely related solid ${ }^{4} \mathrm{He}$.

In Table 1 are shown the exchange frequencies for 2, 3 and 4 atom exchanges in hcp ${ }^{4} \mathrm{He}$. For these calculations, we use an imaginary time step of $0.0125 K^{-1}$ and a value of imaginary time of $\beta=$ $2 K^{-1}$ (160 time slices). For most of the exchanges we find an exchange time $\gamma_{p}=0.3 K^{-1}$. We have studied systems with 48 and 180 atoms; the larger value is necessary for convergence of the exchange frequencies as is shown in the table. The values are quite small e. g. $J_{2} \sim 3 \mu K$ at melting density. We note that in an hcp crystal, there are often several alternative ring exchanges of $\mathrm{n}$ atoms[13]. For example, one can have a 2 particle near-neighbor exchange in the basal plane $n$ or out of the basal plane $n^{\prime}$. Similarly a 3 -body ring exchange can either enclose atoms in nearby planes $T$ or not $T^{*}$. The frequencies for these various alternatives can differ by an order of magnitude.
The small cyclic exchanges in Table 1 are quite different from the long exchanges needed to get a supersolid. Accordingly, we have performed exchange calculations of 50 different exchanges involving from 5 to 10 atoms. All exchanges are between nearest neighbors, since calculations show that next-nearest exchanges are much less probable. We obtain accuracies on the order of $5 \%$ for the 5-particle exchanges and $10 \%$ for the 9 particle exchanges. More than half of the exchanges we studied involve winding around the cell boundaries, important because they are representative of the type of exchanges in a supersolid.

Fig. 1 shows the results of calculations of the frequency of the simplest winding exchanges: straight line exchanges in the basal plane. These calculations were done in an hcp supercell by varying the number of unit cells in the $\mathrm{x}$-direction and keeping a fixed width in the $y$ and $\mathrm{z}$ directions. We find that the exchange frequencies decrease exponentially with the length of the exchange with an exponent of $\alpha=2.64$ near the melting volume 21.04 $\mathrm{cm}^{3}$, and $\alpha=3.14$ at the volume $19.01 \mathrm{~cm}^{3}$ corresponding to $P \sim 60$ bars.

In order to understand whether the BoseEinstein transition occurred in superfluid ${ }^{4} \mathrm{He}$, Feynman introduced a lattice model [14]. The Feynman-Kikuchi (FK) partition function is:

$Z=Z_{0} \sum_{P} e^{-\alpha \sum_{i=1}^{n_{P}} L_{p_{i}}}$

where the sum is over all closed non-intersecting polygons drawn on a lattice by connecting nearest neighbors. Calculations were done on a cubic 3D lattice, first by approximately by Feynman and Kikuchi and then numerically by Elser[15]. It was found that the superfluid transition occurs as $\alpha$ becomes smaller from a localized state (small cycles) to large cycles for $\alpha_{c} \approx 1.44$. To understand this critical value, consider the free energy of adding a link to an existing loop. Adding a link costs probability $e^{-\alpha}$ but the entropy of the new link is 5 in a cubic lattice; hence $\alpha_{c} \sim \ln (5)=1.6$. The exact critical value is smaller because of the 'selfavoiding' restriction within a cycle and on overlapping cycles. Using the same argument for the hcp lattice gives a critical coupling of $\alpha_{c} \approx 2.3$. Our 


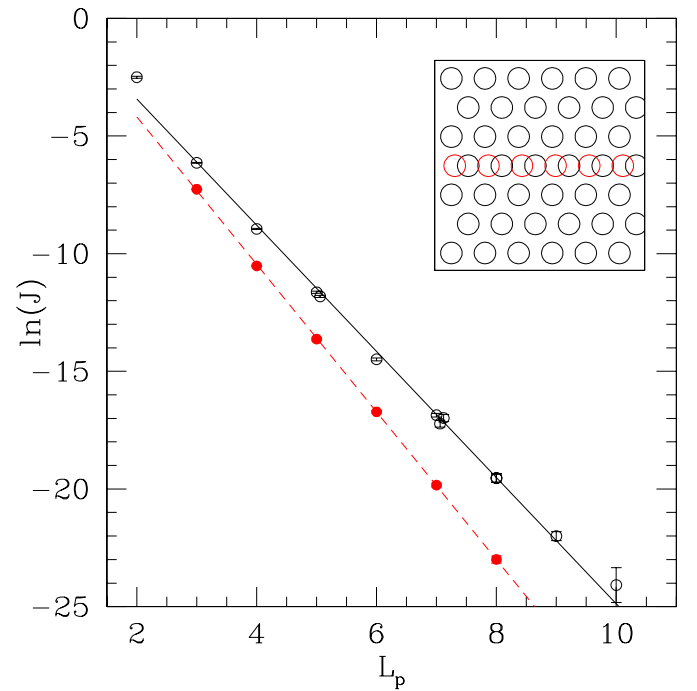

Fig. 1. The exchange frequencies ( $\mathrm{J}$ in $\mathrm{K})$ versus exchange length $L_{p}$ for straight line exchanges in the basal plane that wind around the periodic cell. The inset shows the lattice sites in the basal plane for $L_{p}=6$; the red atoms show the atoms midway through the exchange. The circles with error bars are the $\ln (J)$ at two molar volumes; $21.04 \mathrm{~cm}^{3}$ (black, open circles, solid line) and $19.01 \mathrm{~cm}^{3}$ (red, solid circles, dashed line). The lines are least squares fits.

determined slope in figure 1 is larger than this but not by much!

However, we need a more realistic model than the FK model to take into account more details of the geometry of the exchange than just the number of exchanging atoms; note for example the large differences between the 2 types of nearest neighbor 4-atom exchanges, a parallelogram $K_{p}$ and a square $K_{s q}$ in the Table. We assume that it is only the internal geometry of the exchange that matters[16]; the detailed arrangement of the neighboring spectator atoms is much less important. In particular, we assume the the log of the exchange frequency is a sum over the internal angles $\theta_{k}$ of the exchange:

$J_{p}=J_{0} \exp \left[-\sum_{k=1}^{p} \alpha\left(\theta_{k}\right)\right]$.

In an hcp lattice there are 7 possible angles between two nearest neighbor displacement vectors (neglecting possible dependence on motion with respect to the basal plane), but $\theta=0$ only oc-

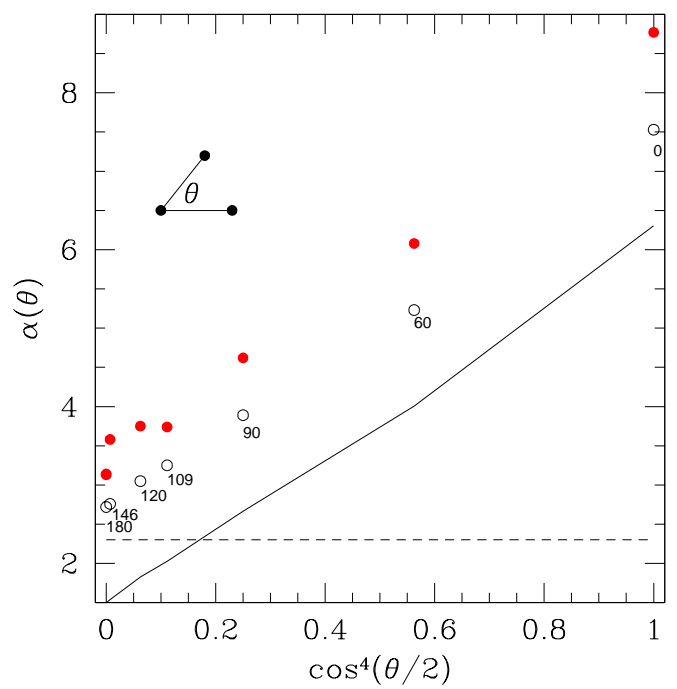

Fig. 2. The fitted angular dependance of the action vs. $\cos ^{4}(\theta / 2)$ at the melting density, 25 bars (open circles). The angle is the interior angle of a vertex as shown. The fits were done using 50 different exchanges in a 180 atom cell. The solid red circles correspond to a pressure 60 bars. The two lines show a critical supersolid Hamiltonian with (solid line) and without (dashed line) angular dependance.

curs in the pair exchange which we neglect since it does not contribute to the superfluid density. We determine the values of the 7 parameters by fitting to the PIMC exchange frequencies $J_{p}$. Good fits are obtained; the model predicts the exchanges frequencies with an accuracy of about $20 \%$. (Note that the data base does not include the small exchanges in table 1 . The frequencies for small exchanges are more sensitive to the neighbors.) There is a strong preference for exchanges that proceed in a straight line versus ones which have sharp angles; for them the incoming and outgoing particles are more likely to collide. We find that the fitting coefficients depend linearly on $\cos ^{4}(\theta / 2)$ so that in the generalized F-K model we assume:

$J_{p}=J_{o} \exp \left[-\alpha L_{p}-\alpha^{\prime} \sum_{i=1}^{n_{p}} \cos ^{4}\left(\theta_{i} / 2\right)\right]$

With the improved model, we can return to the question of whether solid ${ }^{4} \mathrm{He}$ is a supersolid. Because of the directionality of exchange, the probability of retracing is small and we can ignore the self intersections, allowing us to find the probabil- 


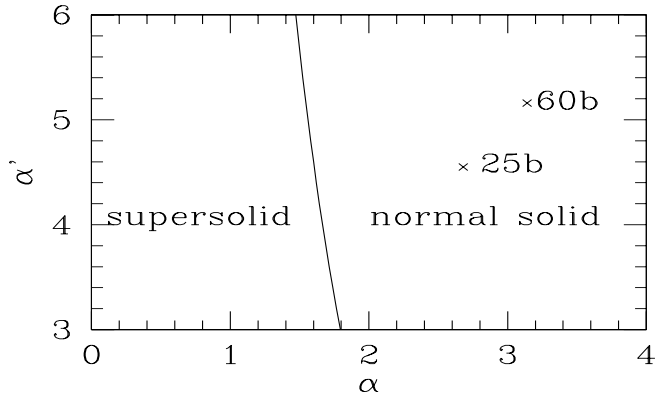

Fig. 3. Phase diagram of the generalized Feynman-Kikuchi model. The 2 points shown are for a density near melting and at high pressure.

ity of long exchanges by formulating it as a Markov process: the diffusion of a particle in an hcp lattice. Note that on the basal plane, exchanges can go straight $(\theta=180)$, while an exchange out of the basal plane must zig-zag $(\theta=146)$, reducing the exchange probability, thus the diffusion is anisotropic. Then, an atomic displacement only depends on the previous atom's displacement, not on the position in the lattice. (Because the hcp lattice has a basis, we have to label the displacements consistently on the A and B planes so that the transition probabilities are independent of the plane.) Let the (un-normalized) probability of a given displacement vector after $n$ steps be $\Pi_{i}^{(n)}$ where $i$ refers to one of the lattice directions. Then it will satisfy the master equation

$\Pi_{j}^{(n+1)}=\sum_{i} \Pi_{i}^{(n)} e^{\alpha\left(\theta_{i}-\theta_{j}\right)}$

The probability for long exchanges will correspond to the maximum eigenvalue $\lambda$ of its stationary state:

$\sum_{i} \Pi_{i} e^{\alpha\left(\theta_{i}-\theta_{j}\right)}=\lambda \Pi_{j}$

By symmetry $\Pi_{i}$ can only depend on whether the direction is in the basal plane, $\Pi_{1}$, or out of the basal plane, $\Pi_{2}$. Define the partial sum $D_{i, j}=$ $\sum_{j} e^{-\alpha\left(\theta_{i}-\theta_{j}\right)}$ where $(i, j)$ are either in the basal plane (1) or out of it (2), e.g. $D_{11}$ is the sum of the probability of both vectors being in the basal plane). The eigenvalue $\lambda$, is the solution to the secular equation:

$\left(D_{11}-\lambda\right)\left(D_{22}-\lambda\right)-D_{12}^{2}=0$.
Table 2

Parameters for the generalized F-K exchange model, Eq. 5 as determined by fitting to PIMC determined exchange frequencies.

\begin{tabular}{|c|c|c|c|c|c|}
\hline $\mathrm{v}\left(\mathrm{cm}^{3}\right)$ & $\mathrm{P}(\mathrm{bar})$ & $J_{0}(\mathrm{~K})$ & $\alpha$ & $\alpha^{\prime}$ & $\lambda$ \\
\hline 21.04 & 25 & 7.2 & 2.64 & 4.8 & 0.31 \\
19.01 & 60 & 8.0 & 3.14 & 4.8 & 0.25 \\
\hline
\end{tabular}

$\lambda=\left(D_{11}+D_{22}\right) / 2+D_{12}\left[1+y^{2}\right]^{1 / 2}$

where $y=\left(D_{22}-D_{11}\right) /\left(2 D_{12}\right) \approx-0.023$. Putting in the model parameters, we find $\lambda=0.303 \pm 0.005$. The probability of having an exchange of length $n$ will equal a prefactor times $\lambda^{n}$, hence, since $\lambda<1$, our PIMC results imply that solid ${ }^{4} \mathrm{He}$ will have only localized exchange and thus cannot be a supersolid. Since $y$ is small, the diffusion in and out of the basal plane are similar. Self-intersections and the presence of other exchange cycles will further decrease the probability of long exchange cycles and increase the critical value of $\lambda$. (To estimate the effect of self-intersections quantitatively, we performed random walks on the hcp lattice and counted non-intersecting walks. This line is shown in Fig. 2.) As seen on Fig. 3 the estimated value of $\lambda$ is much less than what is needed to allow for a supersolid. In addition, there is no indication that long exchanges become prevalent near melting.

One assumption made in the calculations is that long exchanges are correctly being treated by the method to calculate $J$. We find that the exchange time is roughly independent of the number of exchanging atoms, implying that an exchange of $L_{p}$ atoms happens by all of the atoms simultaneously sliding over to their new positions. As $L_{p}$ increases, such a mechanism seems unlikely. There would be much more phase space for the exchange path if different portions of a long path exchanged at different times.

To understand the mechanism for long exchanges, we have done special simulations of them. Though it is not possible to calculate the frequency by the existing algorithm, it is straightforward to sample long exchange paths. In particular, we have studied the exchanges shown in fig. 1 , where $L_{p}$ atoms slide along the basal plane in the x-direction, where $10 \leq L_{p} \leq 50$. The boundary conditions in imaginary time are $R(0)=Z$ 
and $R(\beta)=P Z$ where $P$ is a cyclic exchange of atoms along a straight line. (The cell has a fixed extent in the $\mathrm{y}$ and $\mathrm{z}$-direction.) We do single particle updates on all the atoms using the third level bisection procedure. Because of the ergodic problem, the initial exchange will never decay, so we can study it at will. In particular, we are interested in whether a long exchange maintains the instanton character referred to above, or whether it becomes delocalized in time.

In order to quantify the exchange process, we define a reaction coordinate of each atom involved in the exchange as $\eta_{i}(t)=-1 / 2+\left(x_{i}(t)-x_{i}(0)\right) / a$ where $a$ is the nearest neighbor distance and $x_{i}(t)$ is the $x$-coordinate of atom $i$ at imaginary time $t$. The reaction coordinate is defined so that $\eta_{i}(0)=$ $-1 / 2$ and $\eta_{i}(\beta)=1 / 2$. Fig. 4 is a gray scale image of the reaction coordinates for a typical path with $L_{p}=40$. One sees a fairly sharp division between the initial lattice sites (in black on the left) and the final lattice position (light color on the right). We define the exchange time $e_{i}$ for a given atom as the solution to $\eta_{i}\left(e_{i}\right)=0$. In case of multiple solutions we find the solution where the neighbors atoms are also exchanging. The exchange times for the path in fig. 4 are shown as dots and connected with a solid line.

The resulting curve of exchange times can be used to characterize the exchange process. Nearly vertical portions, represent instantons. In the figure, 16 atoms (labelled on the y axis as 27-40 1-3 ) are such a segment and the 5 atoms (8-12) another. However, in long exchanges, such segments are broken by an interstitial vacancy (i-v) formation, a subsequent pinning of the interstitial and diffusion of the vacancy. An example is atom 16 followed by the diffusion of the vacancy (atoms 17-26). The exchange rarely proceeds by a single $\mathrm{i}-\mathrm{v}$ event. The jagged appearance of the exchange curve is confirmation that vacancies and interstitials are strongly bound and cannot be separated by more than a few lattice spacings. This is different than the creation of a single i-v pair that is discussed in Prokof'ev and B. Svistunov[8]. In the path represented in fig. 4 , at least $3 \mathrm{i}-\mathrm{v}$ fluctuations and 4 or more instantons are present. By forcing the long exchange, we create multiple $\mathrm{i}-\mathrm{v}$ pairs as in a recently proposed supersolid mechanism[18]. However, such i-

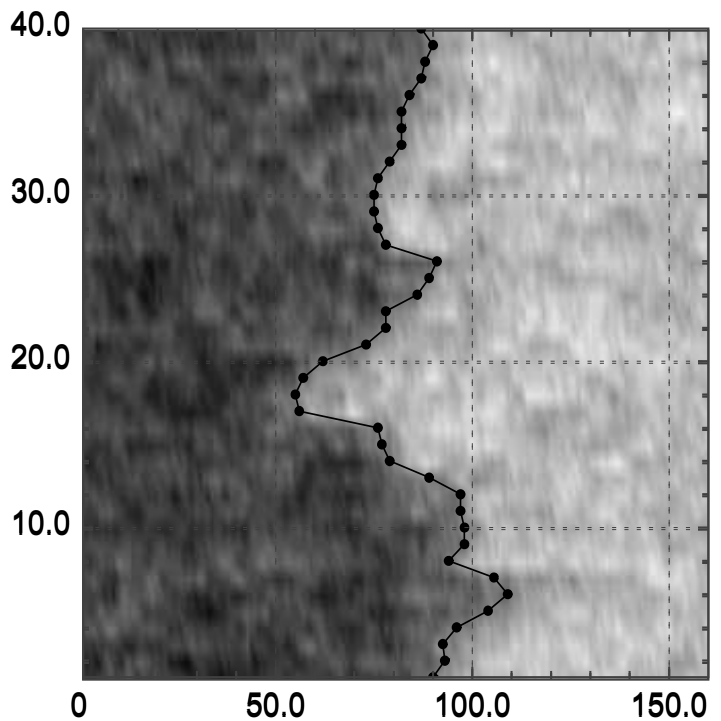

Fig. 4. Gray scale image of the reaction coordinate of a winding path containing 40 atoms, as a function of particle number ( $\mathrm{y}$ axis) and imaginary time (x-axis). The darker region on the left side have coordinates closer to the initial lattice sites, the lighter region on the right side is closer to the permuted lattice. The black dots show the reaction time: when each atom is midway between its initial and final positions. A vertical portion of the reaction time curve (such as at the top) represents a group of atoms that move as a unit. Horizontal (or jagged) parts of the curve represent interstitials.

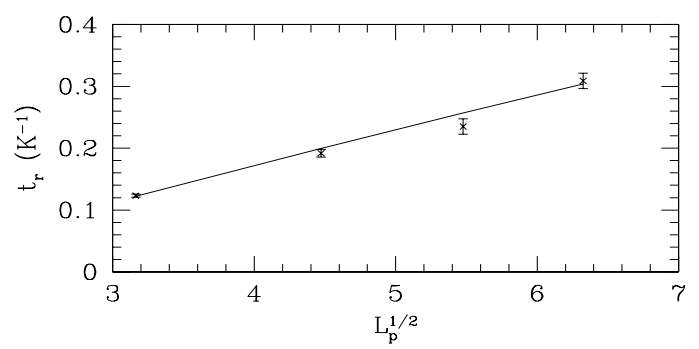

Fig. 5. The "roughness" of the exchange time curve versus the number of exchanging atoms. See the text for the definition of roughness. The solid curve is a calculation of a simple Brownian bridge model of the roughness.

v pairs are not low energy excitations; otherwise they would have been detected in specific heat or other thermodynamic measurements[19].

To quantify this process, we examine the "roughness" of the exchange curve versus the system size. 


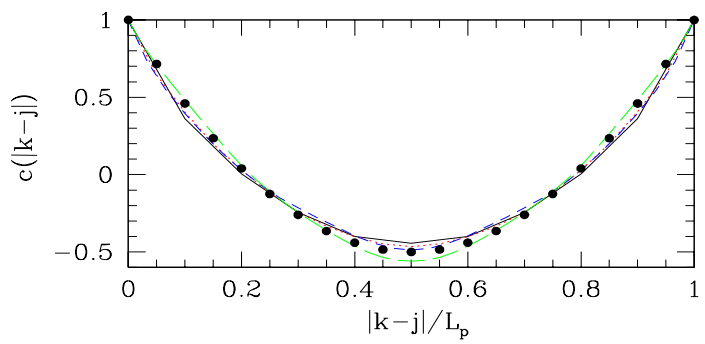

Fig. 6. The auto-correlation (see text) of the exchange time vs separation of atoms. Shown are the results for $L_{p}=10$ (black), 20(red), 30(blue) and 40(green). The curve goes negative for large cycles because it must average to zero and has periodicity $L_{p}$. The dots are analytic results for a Brownian bridge.

We characterize the roughness by first sorting the exchange times and discarding the largest and smallest $L_{p} / 10$ values to make the statistics more robust. The roughness is then $t_{r}=\left\langle e_{\max }-e_{\min }\right\rangle$. The roughness grows as $L_{p}^{1 / 2}$, see fig. 5. Also shown is the same measure of roughness computed on a Brownian bridge with $L_{p}$ elements (random 1D diffusion returning to the start after $L_{p}$ steps). Figure 6 shows the autocorrelation function $c(k-j)=\left\langle\delta e_{k} \delta e_{j}\right\rangle / t_{r}^{2}$ of the exchange time for systems with $10 \leq L_{p} \leq 40$. The autocorrelation function is very close to that given by that of a Brownian bridge: $c(x)=6 x^{2}-6 x+1$. This is another indication that the exchange time is a random diffusion process. Hence, for long exchanges, the exchange time should grow as $L_{p}^{1 / 2}$, because of the creation of a i-v pairs every 5 to 10 atoms. The decay rate of the exchange frequency will still be exponential: $J=\exp \left(-\alpha L_{p}\right)$ since such a law results from both an instanton process and the creation of multiple i-v pairs.

In summary, PIMC-computed exchange frequencies for hcp solid ${ }^{4} \mathrm{He}$ show that only localized exchanges will be present and thus should not exhibit the property of nonclassical rotational inertia. We find no indication that long cycles behave differently than short cycles. In a separate study, we modelled ${ }^{4} \mathrm{He}$ absorbed in Vycor[17] and found that a persistent liquid layer picture could explain the experimental findings. To explain the bulk ${ }^{4} \mathrm{He}$ experiment one must look for other mechanisms, either non-equilibrium dynamical effects or more complicated lattice defects not allowed by the boundary conditions in our simulations.

This work was supported by CNRS and the fundamental physics program at NASA (NAG8-1760). We also thank V. Blancheton and E. Masahiro for running the code that provided data for figs. 3-4.

\section{References}

[1] E. Kim and M. H. W. Chan, Nature 427, 225 (2004); Science 305, 1941 (2004).

[2] A. J. Leggett, Phys. Rev. Letts. 25, 1543 (1970).

[3] D. M. Ceperley and B. Bernu, Phys. Rev. Letts. 93,155303 cond-mat/0409336 (2004).

[4] G. V. Chester, Phys. Rev. A2 256 (1970).

[5] D. Ceperley, G. V. Chester and M. H. Kalos, Phys. Rev. B 17, 1070 (1978).

[6] Hui Zhai and Yong-Shi Wu, cond-mat/0501309

[7] R. A. Aziz et al., Phys. Rev. Letts. 74,1586 (1995).

[8] N. Prokof'ev and B. Svistunov, cond-mat/0409472

[9] D. M. Ceperley, Rev. Mod. Phys. 67, 279 (1995).

[10] D. J. Thouless, Proc. Phys. London 86, 893 (1965). 55, 1 (1983).

[11] D. M. Ceperley and G. Jacucci, Phys. Rev. Letts. 58, 1648 (1987).

[12] B. Bernu and D. Ceperley in Quantum Monte Carlo Methods in Physics and Chemistry, eds. M.P. Nightingale and C.J. Umrigar, Kluwer (1999).

[13] M. Roger, Phys. Rev. B 30 6432(1980).

[14] R. P. Feynman Phys. Rev. 90, 1291 (1953). R. Kikuchi Phys. Rev. 96, 563 (1954).

[15] V. Elser, PhD. Thesis, U. C. Berkeley, (1984).

[16] B. Bernu and D. M. Ceperley, J. Phys. CM 16, 701 (2004).

[17] S. A. Khairallah and D. M. Ceperley, physics/0502039

[18] X. Dai, M. Ma and F.-C. Zhang, cond-mat/0501373

[19] M. W. Meisel, Physica B 178, 121 (1992). 